\title{
Empiricism and Intelligent Design II: Analyzing Intelligent Design
}

\author{
Sebastian Lutz*
}

Draft: $2011-05-12$

\begin{abstract}
If intelligent design (ID) is to compete with evolutionary theory (ET), it must meet the modified falsifiability challenge, that is, make some deductive or probabilistic observational assertions. It must also meet the modified translatability challenge, which it fails if ET makes all the observational assertions of ID, while ID does not make all the observational assertions of ET. I discuss four prominent and surprisingly diverse formulations of ID and show that each either fails one of the two challenges or is analytically false.
\end{abstract}

Keywords: empiricism; intelligent design; empirical significance; empirical content; theism; auxiliary assumptions

\section{Contents}

1 Introduction 1

2 Two challenges 2

3 What is the theory of intelligent design? 3

4 The two challenges to ID $\quad 8$

4.1 The modified falsifiability challenge $\ldots \ldots \ldots \ldots \ldots \ldots \ldots \ldots$

4.2 The modified translatability challenge $\ldots \ldots \ldots \ldots \ldots \ldots \ldots \ldots$

5 Conclusion 12

\section{Introduction}

The current discussion about the theory of intelligent design (ID) as a competitor of evolutionary theory (ET) is logically related to arguments for theism and the question of the empirical significance of theism. This is because the statement 'God exists' may be translated as ' $[\mathrm{T}]$ here exists necessarily a person without a body (i. e., a spirit) who necessarily is eternal, perfectly free, omnipotent, omniscient, perfectly good, and

*Theoretical Philosophy Unit, Utrecht University. sebastian.lutz@gmx.net. Many thanks to Thomas Müller for helpful comments and discussions. 
the creator of all things' (Swinburne 2004, 7), ${ }^{1}$ which entails but is not entailed by 'An intelligent designer exists'. Analogously, 'God created the biological organisms' entails but is not entailed by 'An intelligent designer created the biological organisms'.

Because of the logical relation between theism and ID, two challenges to theism, the falsifiability challenge and the translatability challenge, are also challenges to ID. In this article's companion piece, I have argued that the two challenges have to be modified, and I will shortly review the results in $\$ 2$. In this article, I aim to asses whether ID meets either of the modified challenges by first elucidating a variety of different and incompatible suggested definitions of ID (\$3). Under one very plausible definition, ID succumbs to the modified falsifiability challenge, and this entails that it is analytically false under other definitions ( $\$ 4.1)$. Under yet another definition, ID succumbs to the modified translatability challenge ( $\$ 4.2)$.

\section{Two challenges}

It is a plausible necessary condition for any empirical theory that it make at least some observational assertions. Under the assumption that every theory that makes observational assertions is incompatible with some observation statement, one can therefore challenge ID proponents to name an observation statement with which the theory is incompatible. However, the assumption that is used to justify this challenge is false, and the challenge has to be modified. Instead of demanding falsifiability, one can directly challenge a proponent of ID to spell out ID's deductive or probabilistic observational assertions.

Definition 1. A theory $H$ makes deductive observational assertions if and only if there are honest auxiliary assumptions $A$ and an observation statement $O$ such that

$$
H \wedge A \vDash O \text { and } A \not \models O \text {. }
$$

A set $A$ of auxiliary assumptions is honest if and only if every $S \in A$ is a justified statement, and $A$ also contains every statement on which the justification of $S$ depends.

Definition 2. A theory $H$ makes probabilistic observational assertions if and only if there are honest auxiliary assumptions $A$ and an observation statement $O$ such that

$$
\operatorname{Pr}(O \mid H \wedge A) \neq \operatorname{Pr}(O \mid A) .
$$

$\operatorname{Pr}(O \mid H \wedge A)$ is called the likelihood of $H$ (for $O$ ). These definitions (Lutz 2010, $\$ 9.2 ; 2011, \$ 5.2)$ are intended to avoid the problems of corresponding definitions by Sober $(2008,151 ; 2008,152)$.

The inequality (2) is meant to have a truth value even if one or both of the occurring terms are undefined (Lutz 2011, \$5.2): If both terms are undefined, the inequality is false, if only one of them is undefined, the inequality is true. It is indeed plausible that $H$ makes observational assertions if assuming $H$ makes it impossible to assign a probability to an observation statement that otherwise would be assigned

\footnotetext{
${ }^{1}$ With a further reference to his discussion of the trinity, Swinburne adds in a footnote: "In understanding God as a person, while being fair to the Judaic and Islamic view of God, I am oversimplifying the Christian view."
} 
a probability by the auxiliary assumptions. For if $\operatorname{Pr}(O \mid A)$ is defined, then, given $A$, one must expect a specific regularity of occurrences of $O$. But if $\operatorname{Pr}(O \mid H \wedge A)$ is undefined, one must expect a breakdown of this regularity, and this expectation is plausibly a prediction.

The translatability challenge in its original form amounts to the claim that theism can be replaced by a set of observational statements without loss of cognitive content. The challenge rests on highly controversial assumptions about cognitive content, and is best supplanted by the challenge that a theory cannot replace a competing one if the competing theory is not disconfirmed and makes all the observational assertions of its suggested replacement, while the replacement does not make all the observational assertions of the competing theory.

Definition 3. Theory $H_{1}$ makes all the deductive observational assertions of $\mathrm{H}_{2}$ if and only if for all honest auxiliary assumptions $A$ and all observation statements $O$,

$$
H_{2} \wedge A \vDash O \text { only if } H_{1} \wedge A \vDash O \text {. }
$$

Definition 4. Theory $H_{1}$ makes all the probabilistic observational assertions of $H_{2}$ if and only if for all honest auxiliary assumptions $A$ and all observation statements $O$,

$$
\operatorname{Pr}\left(O \mid H_{2} \wedge A\right)=\operatorname{Pr}(O \mid A) \text { or } \operatorname{Pr}\left(O \mid H_{2} \wedge A\right)=\operatorname{Pr}\left(O \mid H_{1} \wedge A\right) \text {. }
$$

Clearly, a theory that fails the modified falsifiability challenge also fails the modified translatability challenge if it competes with a theory that makes any observational assertions and is not disconfirmed.

\section{What is the theory of intelligent design?}

To determine whether ID fails either of the two challenges, it is necessary to know more or less precisely what the theory of intelligent design is. Sober $(2007,3)$ considers a minimal version of ID:

The single thesis of what I will call mini-ID is that the complex adaptations that organisms display (e. g., the vertebrate eye) were crafted by an intelligent designer.

Since a theory is more likely to fail either of the two challenges the weaker it is, miniID is unfairly minimal: According to Hartwig and Meyer (1993, 160), for example, "the concept of intelligent design predicts that complex information, such as that encoded in a functioning genome, never arises from purely chemical or physical antecedents". While Sober only refers to complex adaptations of organisms, Hartwig and Meyer refer to any kind of complex information. The technical term in the ID literature is 'complex specified information' ('CSI'). ${ }^{2}$ Thus ID should be taken to be the statement that the objects with CSI were caused by an intelligent designer.

According to Sober $(2008,148$; cf. 2008, 130; 2007, 4), "it is perfectly clear" that ID entails the existence of objects with CSI (in his version of ID, the existence of complex adaptations). Whether this is so depends on how the definite article is

\footnotetext{
${ }^{2}$ In this section, it suffices to rely on an intuitive notion of CSI: New York, brains, and computer chips have it, Kasimir Malewitsch's "Black square”, rocks, and sweat do not.
} 
interpreted. Under a suggestion by Sharvy $(1980,615)$, who follows Russell (1905), Sober's version of ID is to be paraphrased as "There is a collection of objects with CSI such that all collections of objects with CSI are its parts, and this collection of objects with CSI was caused by a designer". If there are no objects with CSI, there cannot be a collection of them, so that in this paraphrase, ID entails the existence of objects with CSI. The same holds for a suggestion by Brogaard (2007, 164), which leads to the paraphrase "There are objects with CSI that were created by a designer and all objects with CSI are some of them". Brushing over the subtleties of the natural language formulation, both paraphrases can be formulated as 'There are objects with CSI and all objects with CSI are caused by an intelligent designer. ${ }^{3}$ Letting, for any $x$ and $y$, $\ulcorner D y\urcorner$ stand for $\ulcorner y$ is an intelligent designer $\urcorner$ and $\ulcorner C y x\urcorner$ stand for $\ulcorner y$ caused $x\urcorner$, this leads to the following paraphrase of Sober's definition of ID:

ID 1. There are objects with CSI, and for all objects $x$ with CSI,

$$
\exists y(D y \wedge C y x) .
$$

Russell's definition of the definite article is not the only plausible one. According to Strawson, the definite article indicates that the existence of the objects in its scope is presupposed, that is, a background assumption (cf. Ludlow 2009, \$4.2). Assuming that 'CSI' is an observational concept, 'There are objects with CSI' is justified by observations. The statement can thus be an honest auxiliary assumption, and assuming that the statement is in $A$, ID can be defined as follows:

ID 2. For all $x$ with CSI,

$$
\exists y(D y \wedge C y x) .
$$

ID 1 entails but is not entailed by ID 2 . Hartwig and Meyer $(1993,160)$ arguably have ID 2 in mind when they claim that ID entails that CSI never arises from purely chemical or physical antecedents without claiming that ID entails the existence of objects with CSI. Dembski (2006, emphasis removed) states that "[p]roponents of intelligent design, known as design theorists, $[\ldots]$ claim that a type of information, known as specified complexity, is a key sign of intelligence." If 'key sign' means 'sufficient condition', then proponents of intelligent design claim that all complex specified information is caused by an intelligent designer, that is, ID 2 .

Sober's version of ID is not the only one suggested. Some are obviously wrong: Dembski and Wells $(2008,3)$ define intelligent design as the "study of patterns in nature that are best explained as the product of intelligence", which would make ID a field of study like physics, not a theory like Newtonian mechanics. As a field, it would therefore not make any assertions and succumb to the modified falsifiability challenge. Other definitions differ radically in their logical structure from ID 1 and ID 2: According to the Discovery Institute (N. N. 2010a), ${ }^{4}$

$[\mathrm{t}]$ he theory of intelligent design holds that certain features of the universe and of living things are best explained by an intelligent cause, not an undirected process such as natural selection.

\footnotetext{
${ }^{3}$ The substitution of 'caused' for 'created' is meant to align the paraphrase with the definitions of ID proponents discussed below.

${ }^{4}$ I thank Casey Luskin for helpful discussions about this definition.
} 
Two sections below (N. N. 2010b), the "certain features" of the universe and living things are further specified:

Intelligent design begins with the observation that intelligent agents produce complex and specified information (CSI). Design theorists hypothesize that if a natural object was designed, it will contain high levels of CSI. Scientists then perform experimental tests upon natural objects to determine if they contain complex and specified information. One easily testable form of CSI is irreducible complexity, which can be discovered by experimentally reverse-engineering biological structures $[\ldots]$.

Thus it is claimed that, starting from the observed relation between intelligent agents and CSI, ID theorists conjecture that all natural objects caused by an intelligent designer contain CSI and go on to search for natural objects with CSI. ${ }^{5}$ Note that the ID theorists only conjecture that in our world, the material implication from an intelligent cause of an object to the occurrence of CSI in that object is true, which is a statement of factual co-occurrence. It is another step to the claim that an intelligent cause explains the occurrence of CSI (see the discussion in connection with ID 3 and $\$ 4.1)$. The further assumption that undirected processes do not explain CSI or are unlikely to do so (critically discussed, for example, by Häggström (2007a,b)) then leads to the Discovery Institute's definition of ID.

Thus the Discovery Institute's definition can be paraphrased as follows:

The theory of intelligent design holds that those features of the universe and of living things with CSI are best explained by an intelligent cause, not an undirected process.

Since living things are in the universe, their mention is redundant. ${ }^{6}$ Since an intelligent cause (a designer) is claimed to explain features with CSI best, it is claimed to explain the features better than any process without an intelligent cause. In the following I will call any such process 'undirected'. Finally, the explanation of a feature $F$ by a cause $C$ only makes sense if $C$ is the cause of $F$. Identifying 'being caused by' and 'being the result of', the definition can once more be paraphrased as

The theory of intelligent design holds that for all $x$ with CSI, the statement that $x$ was caused by an intelligent designer explains the CSI of $x$ better than any statement according to which $x$ was caused by an undirected process.

The further discussion of the Discovery Institute's definition will rely on the explication of 'explanation'. This is a notoriously difficult subject, and for this reason, few if any scientific theories rely on the concept as a primitive. ${ }^{7}$ For a definition of

\footnotetext{
${ }^{5}$ This is somewhat puzzling because the conjecture does not seem to be inductively supported by the observations and, in fact, false: Even many objects intentionally produced by intelligent agents, for example the "Black Square", anvils, and (sometimes) sweat, do not show CSI. Note that the conjecture's converse is just ID 2, and thus cannot be assumed to be inductively supported without begging the question (cf. Sober 2008, 176).

${ }^{6}$ Logically redundant, that is. Mentioning living things puts an emphasis on the main application of ID, irreducible complexity in organisms.

${ }^{7}$ Of course, it is claimed of many theories that they explain some phenomenon or other, but that does not mean that the theory itself uses the concept of explanation.
} 
ID, there are good reasons not to connect explanation to the psychological concept of understanding, for one because then a good explanation for one person is not always a good explanation for another who may, for example, lack specific background knowledge. Furthermore, if explanation is connected to understanding, ID becomes a theory that is at least in part about human psychology rather than objects with CSI.

The classic non-psychological explication of 'explanation' relies on deductive inference (cf. Hempel 1965), so that, roughly, given our background assumptions $A, H_{1}$ explains $O$ better than $H_{2}$ explains $O$ if $H_{1} \wedge A \vDash O$ and $H_{2} \wedge A \not \models O$. Likelihoods provide another way to explicate what it means to be a better explanation, by stipulating that $H_{1}$ explains $O$ better than $H_{2}$ if $\operatorname{Pr}\left(O \mid H_{1} \wedge A\right)>\operatorname{Pr}\left(O \mid H_{2} \wedge A\right)$. Using the abbreviations from ID 1 and further letting, for any $x,\left\ulcorner{ }^{C S I}{ }_{x}\right\urcorner$ stand for a description of the CSI of $x$, the Discovery Institute's version of ID is captured by

ID 3. Given our background assumptions $A$, for all $x$ with CSI and any undirected process $u$,

$$
\exists y(D y \wedge C y x) \wedge A \vDash \operatorname{CSI}_{x} \text { and } C u x \wedge A \not \models \operatorname{CSI}_{x}
$$

or

$$
\operatorname{Pr}\left(\operatorname{CSI}_{x} \mid \exists y(D y \wedge C y x) \wedge A\right)>\operatorname{Pr}\left(\operatorname{CSI}_{x} \mid C u x \wedge A\right) .
$$

Background assumptions are almost always needed to assign probabilities to or infer observation statements, and background assumptions should be honest (Lutz 2011, \$\$3.2, 5.2), which I will assume in the following.

ID 3 states a logical or probabilistic relation between design and CSI relative to our background assumptions. This makes ID 3 a rather odd theory: It is not a statement that in fact relates to specific observable phenomena as a matter of logic or probability theory, but it postulates that some other statement does so relate.

In an elaborate discussion of ID, Monton $(2009, \$ 2)$ suggests multiple improvements to the Discovery Institute's definition that also avoid the problematic concept of explanation. He conjectures that ID proponents "wouldn't take much solace in the knowledge that their appeal to an intelligent cause is the best explanation, if we've established that their explanation is a false one". Monton (2009, 38, emphasis removed) therefore suggests a preliminary improvement:

The theory of intelligent design holds that certain features of the universe and of living things are the result of an intelligent cause, not an undirected process such as natural selection.

Keeping in mind that the certain features are those with CSI and that a feature cannot be the result of both an intelligent cause and an undirected process, this paraphrase amounts to ID 2. Since ID 2 differs radically from ID 3 in its logical structure, the justification for this modification is somewhat wanting.

In "a bit of charitable speculation", Monton $(2009,38)$ suggests that ID proponents speak of an intelligent designer as the best explanation because one of the "key ideas behind intelligent design is that their theory is scientific, and one can get scientific evidence for the existence of the intelligent designer". Therefore "the actions of the intelligent cause [must not be] completely hidden from us". Monton (2009, 38, emphasis removed) thus suggests another preliminary definition (*):

The theory of intelligent design holds that certain features of the universe and of living things provide evidence for the existence of an intelligent 
cause, and provide evidence against the doctrine that the features are the result of an undirected process such as natural selection.

The exact nature of ID then depends on how 'providing evidence for' is explicated.

Monton $(2009,100)$ assumes that the evidential relationship is to be explicated by probability theory, and in probabilistic terms, a theory $H$ is confirmed by an observation $O$ given the auxiliary assumptions $A$ if and only if $\operatorname{Pr}(H \mid O \wedge A)>$ $\operatorname{Pr}(H \mid A)$ and disconfirmed if and only if $\operatorname{Pr}(H \mid O \wedge A)<\operatorname{Pr}(H \mid A)$ (Howson and Urbach 1993, \$7a). This leads to

ID 4. Given our background assumptions $A$, for all $x$ with CSI and any undirected process $u$,

$$
\operatorname{Pr}\left(\exists y(D y \wedge C y x) \mid \operatorname{CSI}_{x} \wedge A\right)>\operatorname{Pr}(\exists y(D y \wedge C y x) \mid A)
$$

and

$$
\operatorname{Pr}\left(C u x \mid \operatorname{CSI}_{x} \wedge A\right)<\operatorname{Pr}(\operatorname{Cux} \mid A)
$$

Of course, there are other conceptions of confirmation. Sober $(2008, \$ 1.3)$, for example, argues that evidential support should be construed contrastively, so that an observation statement $\left\ulcorner\mathrm{CSI}_{x}\right\urcorner$ confirms $\ulcorner\exists y(D y \wedge C y x)\urcorner$ and disconfirms $\ulcorner C u x\urcorner$ relative to each other if and only if $\operatorname{Pr}\left(\operatorname{CSI}_{x} \mid \exists y(D y \wedge C y x \wedge A)>\operatorname{Pr}\left(\operatorname{CSI}_{x} \mid C u x \wedge A\right)\right.$. This explication of confirmation and disconfirmation leads to the second disjunct, equation (8), of ID 3.

Monton's second modification (*) of the Discovery Institute's definition brings his own definition again closer to the original (and to ID 3), since ID again claims a probabilistic relation between a designer and CSI. His argument for the second modification may indeed provide the reason for the Discovery Institute's definition of ID, but it is an odd one nonetheless: Rather than trying to develop a theory involving a designer that is scientific, that explains something, or that can be supported by evidence, ID theorists, according to Monton's argument, simply postulate that for any $x$ with CSI, $\ulcorner\exists y(D y \wedge C y x)\urcorner$ is scientific, explains something, or is supported by evidence. I will discuss the implications of this move in $\$ 4.1$.

Monton $(2009,38)$ charges that both the Discovery Institute's definition of ID and his preliminary definition (*) are trivially true, because in a crucial point, Monton's interpretation of the Discovery Institute's definition of ID differs from the one assumed in ID 3 and ID 4. This becomes clear when Monton $(2009,72)$ discusses the assertions of ID:

I would say that intelligent design proponents are making a prediction: they are claiming that, if one looks, one will find evidence that there is a designer.

I have read 'certain features' in the Discovery Institute's definition as a placeholder for 'instances of CSI'. Monton instead reads 'certain features' as 'some features', so that ID becomes the claim that there are features that are best explained by an intelligent cause, not by an undirected process.

Under this reading, it is indeed indisputably and almost trivially true that there are certain features of the universe that provide evidence for a designer and against the claim that they are the result of an undirected process. The Petronas Towers in 
Kuala Lumpur and Sarah Watson's muscular arms are examples (Monton 2009, 16, 23).

I have argued above for my interpretation, and it also fits better with Hartwig and Meyer's claim about the impossibility of CSI arising from purely physical or chemical antecedents, the central role that CSI and irreducible complexity play in the exposition of ID by Dembski and Wells (2008), and specifically Dembski's claim that "if there is a way to detect design, specified complexity is it" (Dembski 2002, 116). The lengths to which Monton has to go to avoid the almost trivial truth of ID provide another argument against his interpretation. His final definition is this (Monton 2009, 39, emphasis changed):

The theory of intelligent design holds that certain global features of the universe provide evidence for the existence of an intelligent cause, or that certain biologically innate features of living things provide evidence for the doctrine that the features are the result of the intentional actions of an intelligent cause which is not biologically related to the living things, and provide evidence against the doctrine that the features are the result of an undirected process such as natural selection.

Except for the change from a conjunction to the disjunction (here emphasized), all the modifications are attempts at avoiding the almost trivial truth of ID (Monton 2009, 17-26). None of the modifications are necessary under the interpretation defended here, since it does not render ID almost trivially true, and renders the mention of living things in the Discovery Institute's definition redundant. ${ }^{8}$

It is furthermore doubtful that Monton avoids triviality or something close to it. If, for example, the hypothesis of a designer asserts a certain feature $F$ in all organisms, while no undirected process does, the existential quantification allows to include all and only those organisms with feature $F$ in the evidence. If the hypothesis of a designer, but no undirected process, asserts a specific ratio of $F$, the existential quantification allows to pick out a set of organisms that has this very ratio as long as there are enough organisms with and enough organisms without the feature to assemble the set. Therefore, as long as the hypothesis of the designer makes any such assertion, ID is true under Monton's definition. ${ }^{9}$

\section{The two challenges to ID}

The previous section has resulted in four versions of ID. ID 1 is Sober's own clarification of his definition, using Russell's paraphrase of the definite article. ID 1 entails ID 2, which itself is a clarification of Sober's definition that relies on Strawson's paraphrase of the definite article. The Discovery Institute's definition can be clarified as ID 3. Finally, while Monton's final definition of ID is flawed, his preliminary

\footnotetext{
${ }^{8}$ Note that neither ID 3 nor ID 4 exclude the alleged fine-tuning of cosmological constants as evidence for an intelligent designer (which is a focus of Monton's discussion): Dembski (2002, xiii) claims that the fine-tuning is an instance of CSI.

${ }^{9}$ Monton $(2009,35,109)$ also seems to existentially quantify over all designers, as he rather freely chooses the designer's intentions (to let an atom decay at one specific moment or to have as much intelligent life as possible, for example). Strictly speaking, Monton is thus not dealing with the question whether there could be any evidence $\left\ulcorner\operatorname{CSI}_{x}\right\urcorner$ for $\ulcorner\exists y(D y \wedge C y x)\urcorner$, but with the question whether there could be a $y$ such that there could be any evidence $\left\ulcorner\operatorname{CSI}_{x}\right\urcorner$ for $\ulcorner D y \wedge C y x\urcorner$.
} 
definition $(*)$ suggests ID 4 if Bayesianism is used as a criterion of confirmation, and the probabilistic disjunct of ID 3 if likelihoodism is used.

In this section, I will discuss which of these versions of ID meets which of the two challenges discussed in $\$ 2$. For this discussion, I will assume that CSI is an observational concept. This assumption is charitable because either challenge is easier to meet the more terms in a theory are observational, and because CSI is observational only if it is well-defined, which is disputed, for example, by Elsberry and Shallit (2009, $\$ \$ 4-6)$.

\subsection{The modified falsifiability challenge}

According to Swinburne $(2004,7)$, 'God' is, in the three world-religions, synonymous with 'A necessarily existing person without a body who necessarily is eternal, perfectly free, omnipotent, omniscient, perfectly good, and the creator of all things'. This synonymy is therefore a justified auxiliary assumption, which allows to infer from God's existence that all objects, and hence all objects with CSI, are caused by a person (and hence a designer). Any explanation of a phenomenon by this theory is, according to Swinburne (2004, 47ff), a personal explanation, and as such it relies essentially on the intentions and abilities of the designer, in this case God. ID 2 is a personal explanation as well and therefore entails an observation (for example the description of the CSI of some object $x$ ) only in conjunction with two specific auxiliary assumptions. The first states that any designer who caused an object $x$ with CSI has intentions $i_{x}$ and abilities $a_{x}$, that is, with $\left\ulcorner I y i_{x}\right\urcorner$ standing for $\left\ulcorner y\right.$ has intention $\left.i_{x}\right\urcorner$ and $\left\ulcorner B y a_{x}\right\urcorner$ standing for $\left\ulcorner y\right.$ has ability $\left.a_{x}\right\urcorner$ :

$$
\text { For all } x \text { with CSI: } \forall y\left(D y \wedge C y x \rightarrow I y i_{x} \wedge B y a_{x}\right) \text {. }
$$

The second states that a designer who caused $x$ with intentions $i_{x}$ and abilities $a_{x}$ brings about the observation $O_{x}$ :

$$
\text { For all } x \text { with CSI: } \forall y\left(D y \wedge C y x \wedge I y i_{x} \wedge B y a_{x} \rightarrow O_{x}\right) .
$$

If some $x$ has CSI, observation $O_{x}$ then follows because for all $x$ with CSI,

$$
\begin{aligned}
\exists y(D y \wedge C y x) \wedge \forall y(D y \wedge C y x & \left.\rightarrow I y i_{x} \wedge B y a_{x}\right) \wedge \\
& \forall y\left(D y \wedge C y x \wedge I y i_{x} \wedge B y a_{x} \rightarrow O_{x}\right) \vDash O_{x} .
\end{aligned}
$$

For probabilistic assertions, the likelihoods of $\ulcorner\exists y(D y \wedge C y x)\urcorner$ can be given through generalizations of the deductive premises. Instead of claiming that if $x$ was designed by $y, y$ has intention $i_{x}$ and ability $a_{x}$, it is enough to assert that if $x$ was designed, the probability that it was designed by some $y$ with intention $z$ and ability $w$ has the probability $p_{x y z w}$ :

$$
p_{x y z w}=\operatorname{Pr}(D y \wedge C y x \wedge I y z \wedge B y w \mid \exists y(D y \wedge C y x)) .
$$

The second deductive auxiliary assumption (12) can be relaxed to the claim that if a designer $y$ of $x$ has intention $z$ and ability $w$, then an observation $O$ has probability $q_{x y z w O}$ :

$$
q_{x y z w O}=\operatorname{Pr}(O \mid D y \wedge C y x \wedge I y z \wedge B y w) .
$$


The probability for $O$ for any $x$ with CSI can then be written as ${ }^{10}$

$$
\operatorname{Pr}(O \mid \exists y(D y \wedge C y x))=\sum_{y z w} p_{x y z w} q_{x y z w O} .
$$

In the theistic theory, the abilities of the designer are known within the precision of natural language: God is omnipotent. The intentions of God are also known within the precision of the word 'good'. ${ }^{11}$ ID 2 differs from the theistic theory in this respect: The designer who caused an object with CSI is only specified as being intelligent. Leaving the problem of the level of intelligence aside, Sober (1999, 62f; 2007,$6 ; 2008, \$ 2.12, \$ 2.15$ ) argues in a number of ways that the intentions of the designer are unknown. Among them is his point that the designer could be an extraterrestrial, which would give no indication about the intentions. But 'designer' is even less specific than 'extraterrestrial designer', and thus would give even fewer indications of the intentions (Sober 1999, 65f). And sometimes, the problem of evil is solved by claiming that the intentions of God are unknowable. This answer entails that the intentions of a less specific designer are also unknowable (Sober 2008, \$2.17). Sober $(1999,74$, n. 25; 2007, 6; 2008, 128, 154, n. 29) also gives lists of proponents of ID who state that the intentions of the designer cannot be known.

In general, for any designer with the intentions and the abilities to do something, one can without inconsistency think of another designer without those intentions or abilities. Thus the first auxiliary assumption (11) of the deductive assertion of $O_{x}$ (13) is unjustified for any intention and ability, with (arguably) one exception. If one observes $O_{x}$ for some $x$, then one can plausibly infer that if there is a designer of $x$, the designer had the intention to bring about $O_{x}$. With the further plausible assumption that having the intention and ability to bring about $O_{x}$, a designer would bring about $O_{x}$, one can infer $O_{x}$. But then, for the auxiliary assumptions to be honest, they have to contain assumption $O_{x}$ if they contain the first auxiliary assumption. Therefore, ID 2 in conjunction with honest auxiliary assumptions entails $O_{x}$ only if the auxiliary assumptions alone already entail $O_{x}$, and thus ID 2 makes no deductive observational assertions.

ID 2 makes a probabilistic assertion, that is, assigns a probability to some observation $O$, only if the sum (16) is defined. The sum in turn is defined only if the conditional probabilities (14) and (15) are defined, again because ID 2 relies on personal explanations. But analogously to the deductive case, for any designer who would have some intention and ability with one probability, one can, without flouting the laws of probability, think of another designer who has that intention and ability with any other probability. Thus the first auxiliary assumption (14) is undefined for almost all intentions and abilities. Similar to the deductive case, the exceptions are those cases in which observation $O$ can be assigned a probability $s$ independently of assuming a designer. For some $O$ and $x$, one can then maybe justify the assumption that if a designer caused $x$, the designer must have had the intention and ability to bring about $O$ with probability $s$. (It is here not obvious what values the probabilities in the two auxiliary assumption have to have, only that the sum of their products has to be $s$ for $O$.) But if $\operatorname{Pr}(O)=s$ is used to justify the auxiliary

\footnotetext{
${ }^{10}$ The condition in equation (15) for term $q_{x y z w O}$ does not need to contain the conjunct $\ulcorner\exists y(D y \wedge$ $C y x)\urcorner$, as this is entailed by $\ulcorner D y \wedge C y x\urcorner$ for any $y$.

${ }^{11}$ This assumes that 'good' has its usual meaning in theistic statements. The contrary assumption lead to the original falsifiability challenge (Flew 1950, 258f).
} 
assumptions, it has to be in the auxiliary assumptions as well if they are honest. Thus, ID 2 only assigns probability $s$ to $O$ if the auxiliary assumptions do, too. Therefore ID 2 makes no probabilistic observational assertions either, and thus succumbs to the modified falsifiability challenge.

As already noted, the problem with ID 3 and ID 4 is that, rather than make observational assertions, they postulate deductive or probabilistic relations between the existence of an intelligent designer and observations. Given the results so far, one can see that the postulates are, in fact, false: ID 3 is true only if, given our background assumptions $A$, equation (7) or equation (8) is true for every $x$ with CSI. Equation (7) states that for each $x,\ulcorner\exists y(D y \wedge C y x) \wedge A\urcorner$ entails a description of the CSI of $x$, while $\ulcorner U x \wedge A\urcorner$ does not. But ID 2 makes no observational assertions for any honest auxiliary assumptions, and for each $x$ with CSI, $\ulcorner\exists y(D y \wedge C y x)\urcorner$ is a logically weaker claim than ID 2. Since CSI $_{x}$ is assumed to be an observation statement, and our background assumptions are specific honest auxiliary assumptions, $\ulcorner\exists y(D y \wedge C y x)\urcorner$ therefore asserts no description of the CSI of $x$. Hence equation (7) is false for all $x$, and ID 3 is false unless equation (8) is true for all $x$. This holds only if for all $x$ with CSI, the probability for the CSI of $x$ given $\ulcorner\exists y(D y \wedge C y x)\urcorner$ is higher than its probability given any undirected process $u$, and thus specifically ET. Sober (2008) has argued extensively that the complex adaptations we observe can be explained by ET, and thus are certainly not less probable under the assumption of ET than they are given our background assumptions. Therefore equation (8) is true only if the probability for the CSI of $x$ given ID 2 is higher than given our background assumptions. But again, since ID 2 and thus $\ulcorner\exists y(D y \wedge C y x)\urcorner$ make no observational assertions, this is false. ID 3 is therefore analytically false, because it postulates an inferential relation that does not exist.

ID 4 is true only if all undirected processes, and thus also ET, are disconfirmed and $\ulcorner\exists y(D y \wedge C y x)\urcorner$ is confirmed by each $x$ with CSI given our background assumptions. Sober $(2007,2008)$ has documented at length that ET has not been disconfirmed by each such $x$ and thus ID 4 is false independently of the status of $\ulcorner\exists y(D y \wedge C y x)\urcorner$ because equation (10) is false. But ID 4 would be wrong even if ET were disconfirmed by each $x$ with CSI: Purportedly, there are objects for which the designer cannot be directly observed and which show CSI. For those objects, $\ulcorner\exists y(D y \wedge C y x)\urcorner$ has to be confirmed by observations other than those of the designer. This holds if and only if equation (9) holds, which is equivalent to

$$
\operatorname{Pr}\left(\operatorname{CSI}_{x} \mid \exists y(D y \wedge C y x) \wedge A\right)>\operatorname{Pr}\left(\operatorname{CSI}_{x} \mid A\right) .
$$

But this inequality cannot be true for the same reason that equation (8) cannot be true, namely because ID 2 makes no observational assertions.

This leaves ID 1, Sober's definition, which as Sober himself has pointed out, meets the modified falsifiability challenge.

\subsection{The modified translatability challenge}

ID 1 clearly makes the deductive observational assertion that there are objects with CSI. But ID 1 is equivalent to the conjunction of this existence claim and ID 2, and I have argued in $\$ 4.1$ that also in conjunction with honest auxiliary assumptions, ID 2 makes no observational assertions. Under the assumption that 'CSI' is a well-defined observational concept, it is a justified and hence honest auxiliary assumption that 
there are objects with CSI. ${ }^{12}$ Therefore ID 1 entails only observation statements that are also entailed by this existence claim, and assigns the same probabilities as this existence claim.

ID 1 fails to meet the modified translatability challenge against ET if ET makes all the observational assertions of ID 1, but ID 1 does not make all the observational assertions of ET. Sober $(2008, \$ 2.9)$ argues that if the existence of objects with CSI (or, in his discussion, of complex adaptations of organisms) is to be explicitly included in ID, it should also be included in ET. This is plausible, as the definite article should be interpreted in the same way, no matter whether the statement is 'The objects with CSI were caused by an intelligent designer' or 'The objects with CSI are the result of natural selection'. This is also in keeping with the description of ID by the Discovery Institute that led to ID 3, because it refers to the intelligent cause and undirected processes in the same way.

With both ID 1 and ET entailing the existence of objects with CSI (let this be claim $E)$, ET makes all the deductive observational assertions of ID 1. Unless there is an observation $O$ such that $\operatorname{Pr}(O \mid E \wedge A) \neq \operatorname{Pr}(O \mid A)$ and $\operatorname{Pr}(O \mid E \wedge A) \neq \operatorname{Pr}(O \mid E \wedge$ ET^ $A$ ), that is, $E$ alone makes a probabilistic observational assertion different from ET, ET also makes all the probabilistic observational assertions of ID 1 . Then, since ET makes observational assertions besides $E$, ID 1 does not meet the modified translatability challenge. But even if $E$ alone makes a probabilistic observational assertion different from ET, ID 1 fails to meet the modified falsifiability challenge against many other statements: $E$ itself makes all the observational assertions of ID 1, and, for example, the claim $E^{\prime}$ that there are organisms with eyes makes more.

\section{Conclusion}

Probably the most important result of the elaboration of ID in $\$ 3$ is that ID is woefully underspecified, leading to different explications with vastly different properties. ID 2 makes no observational assertions and thus fails to meet the modified falsifiability challenge, ID 1 makes fewer observational assertions than ET and in fact the simple claim that there are organisms with eyes, and thus fails to meet the modified translatability challenge. ID 3 and ID 4 are wrong, not because of incorrect observational assertions, but rather because they make incorrect logical or probabilistic claims.

ID is also often formulated with so little precision that even charitable interpreters (which I take Monton and me to be) can arrive at fundamentally incompatible conclusions about its content. An especially egregious example is the description of ID in two of the major textbooks on ID. Beyond defining ID as a field of study, Dembski and Wells $(2008,109)$ claim that ID "neither requires nor excludes speciation-even speciation by Darwinian mechanisms. ID is sometimes confused with a static view of species, as though species were designed to be immutable. [...] ID precludes neither significant variation within species nor the evolution of new species from earlier forms". Davis and an H. Kenyon (1993, 99f), on the other hand, state that ID "means that various forms of life began abruptly through an intelligent agency, with their distinctive features already intact: fish with fins and scales, birds with feathers, beaks, and wings, etc."

\footnotetext{
${ }^{12}$ Again because one can observe many objects that, intuitively, have CSI: Miami, eyes, etc.
} 
Biologists have found overwhelming evidence for common descent, and thus even with this last addition to the superabundance of varieties of ID, the conclusion stays the same: ID fails as a scientific theory, because it is disconfirmed by the evidence, because it makes false inferential claims, because it fails the modified falsifiability challenge, or because it fails the modified translatability challenge. But it fails.

\section{References}

Brogaard, B. (2007). Sharvy's theory of definite descriptions revisited. Pacific Philosophical Quarterly, 88:160-180.

Davis, P. and an H. Kenyon (1993). Of Pandas and People: The Central Question of Biological Origins. Foundation for Thought and Ethics, Richardson, TX, $2^{\text {nd }}$ edition.

Dembski, W. (2006). In defense of intelligent design. In Clayton, P. and Simpson, Z., editors, The Oxford Handbook of Religion and Science, Oxford Handbooks in Religion and Theology. Oxford University Press, Oxford.

Dembski, W. A. (2002). No free lunch: Why specified complexity cannot be purchased without intelligence. Rowman \& Littlefield, Lanham, MD.

Dembski, W. A. and Wells, J. (2008). The Design of Life: Discovering Signs of Intelligence in Biological Systems. Foundation for Thought and Ethics, Dallas, TX.

Diamond, M. L. and Litzenburg, T. V., editors (1975). The Logic of God: Theology and Verification. Bobbs-Merill, Indianapolis, IN.

Elsberry, W. and Shallit, J. (2009). Information theory, evolutionary computation, and Dembski's “complex specified information”. Synthese, 178(2):237-270. Special issue: Evolution and its Rivals, edited by Glenn Branch and James H. Fetzer.

Flew, A. (1950). Theology and falsification. University, 1:1-8. References are to the reprint (Diamond and Litzenburg 1975, 257-259).

Häggström, O. (2007a). Intelligent design and the NFL theorems. Biology and Philosophy, 22(2):217-230.

Häggström, O. (2007b). Uniform distribution is a model assumption. http://www . math. chalmers.se/ olleh/reply_to_Dembski.pdf. Archived at http://www . webcitation.org/5tsNDuhBm.

Hartwig, M. D. and Meyer, S. C. (1993). A note to teachers. In Davis and an H. Kenyon (1993), pages 153-164.

Hempel, C. G. (1965). Aspects of scientific explanation. In Aspects of Scientific Explanation and Other Essays in the Philosophy of Science, pages 331-496. The Free Press, New York.

Howson, C. and Urbach, P. (1993). Scientific Reasoning: The Bayesian Approach. Open Court, Chicago and La Salle, IL. Second edition. Second printing 1996. 
Ludlow, P. (2009). Descriptions. In Zalta, E. N., editor, The Stanford Encyclopedia of Philosophy. The Metaphysics Research Lab, Center for the Study of Language and Information, Stanford University, Stanford, spring 2009 edition.

Lutz, S. (2010). Criteria of empirical significance: A success story. Preprint: http: //philsci-archive.pitt.edu/8423/. Forthcoming.

Lutz, S. (2011). On a contrastive criterion of testability II: The material inadequacy of contrastive testability. Preprint: http://philsci-archive.pitt.edu/8575/. Forthcoming.

Monton, B. (2009). Seeking God in Science: An Atheist Defends Intelligent Design. Broadview Press, Peterborough, ON.

N. N. (2010a). Definition of intelligent design. http://www . intelligentdesign . org/whatisid.php. Archived at http://www . webcitation.org/5tZWKxwwV.

N. N. (2010b). Is intelligent design a scientific theory? http: //www.intelligentdesign.org/whatisid.php. Archived at http: //www. webcitation.org/5tZWKxwwV.

Russell, B. (1905). On denoting. Mind, 14:479-493.

Sharvy, R. (1980). A more general theory of definite descriptions. The Philosophical Review, 89(4):607-624.

Sober, E. (1999). Testability. Proceedings and Addresses of the American Philosophical Association, 73(2):47-76.

Sober, E. (2007). What is wrong with intelligent design? The Quarterly Review of Biology, 82(1):3-8.

Sober, E. (2008). Evidence and Evolution: The Logic Behind the Science. Cambridge University Press, Cambridge.

Swinburne, R. (2004). The Existence of God. Clarendon Press, Oxford, $2^{\text {nd }}$ edition. 